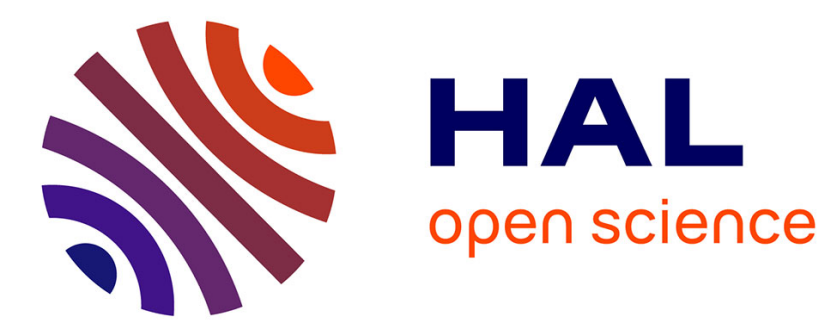

\title{
Stanislas Nordey entre filiation et transmission
}

Marion Denizot

\section{To cite this version:}

Marion Denizot. Stanislas Nordey entre filiation et transmission. Etudes théatrales, 2007, 3 (40), pp.57-66. hal-02871088

\section{HAL Id: hal-02871088 \\ https://hal.science/hal-02871088}

Submitted on 17 Jun 2020

HAL is a multi-disciplinary open access archive for the deposit and dissemination of scientific research documents, whether they are published or not. The documents may come from teaching and research institutions in France or abroad, or from public or private research centers.
L'archive ouverte pluridisciplinaire HAL, est destinée au dépôt et à la diffusion de documents scientifiques de niveau recherche, publiés ou non, émanant des établissements d'enseignement et de recherche français ou étrangers, des laboratoires publics ou privés. 


\title{
STANISLAS NORDEY ENTRE FILIATION ET TRANSMISSION
}

\author{
Marion Denizot \\ L'Harmattan | «Études théâtrales »
}

2007/3 $\mathrm{N}^{\circ} 40$ | pages 57 à 66

ISSN 0778-8738

ISBN 9782930416267

Article disponible en ligne à l'adresse :

https://www.cairn.info/revue-etudes-theatrales-2007-3-page-57.htm

Distribution électronique Cairn.info pour L'Harmattan.

(C) L'Harmattan. Tous droits réservés pour tous pays.

La reproduction ou représentation de cet article, notamment par photocopie, n'est autorisée que dans les limites des conditions générales d'utilisation du site ou, le cas échéant, des conditions générales de la licence souscrite par votre établissement. Toute autre reproduction ou représentation, en tout ou partie, sous quelque forme et de quelque manière que ce soit, est interdite sauf accord préalable et écrit de l'éditeur, en dehors des cas prévus par la législation en vigueur en France. Il est précisé que son stockage dans une base de données est également interdit. 
Deuxième partie

\section{Pratiques théâtrales et milieux populaires}




\section{Marion Denizot}

\section{Stanislas Nordey entre filiation et transmission}

\section{$\ll \mathrm{I}$}

ES ENGAGEMENTS fermes des 'petits-fils' de Vilar. Stanislas Nordey et Olivier Py secouent le cocotier du théâtre public »', écrit Brigitte Salino en 1997. Au-delà de la tension générationnelle tuer le père pour devenir père à son tour -, ce titre introduit la question de l'héritage du théâtre populaire à la fin du XXème siècle, plus de cent ans après son émergence. Quand Stanislas Nordey prend la direction du Théâtre Gérard-Philipe de Saint-Denis (TGP), en 1998, le manifeste qu'il diffuse - «Pour un théâtre citoyen » - revendique l'héritage d'un «théâtre de service public, un théâtre pour tous, à partir des poètes, pour les publics, pour les artistes, aujourd'hui ». Comment comprendre ce nouvel intérêt, à la fin des «années Lang ", pour les fondateurs du théâtre public?

Parce que Stanislas Nordey, en refusant toute approche nostalgique du théâtre populaire, privilégie une mise en pratique de certains de ses dispositifs, au-delà d'une appropriation verbale de bon aloi, l'étude de son parcours forme la matière d'une réflexion sur l'actualisation de l'utopie du théâtre populaire. L'échec de l'expérience du TGP, confronté à une crise financière, contribue paradoxalement à inscrire Stanislas Nordey dans la filiation du théâtre populaire.

Marion Denizot

Maitre de conférences en Études théâtrales, Marion Denizot est directrice du département des Arts du spectacle de l'Université de HauteBretagne-Rennes II, attachée au laboratoire «La présence et l'image » de l'Équipe d'accueil «Arts : pratiques et poétiques».

Diplômée de l'Institut d'Études politiques de Paris, ses travaux portent, d'une part, sur les héritages du théâtre populaire et, d'autre part, sur l'histoire des politiques et le fonctionnement des institutions théâtrales. Elle a publié en 2005 Jeanne Laurent. Une fondatrice du service public pour la culture. 1946-1952, La Documentation Française. En 2006, elle a réalisé pour le compte du Comité d'histoire du ministère de la Culture une étude intitulée Le Plan de développement de l'enseignement musical en Ille-et-Vilaine : une contractualisation atypique pour un schéma référent, dans le cadre d'un programme de recherche coordonné par Philippe Poirrier et Pierre Moulinier. 


\section{«Artiste avant d'être militant » ${ }^{2}$}

Stanislas Nordey acquiert une reconnaissance professionnelle et médiatique dès ses premières mises en scène. Avant d'être porteur d'un projet culturel revendiquant la possibilité d'un "théâtre citoyen », il s'affirme comme un artiste de la radicalité, à l'image de Jean Vilar qui fut d'abord apprécié pour ses mises en scène de Strindberg ou de T.S. Eliot, avant de devenir le représentant du «théâtre, service public ». Formé pendant trois ans au Cours Véronique Nordey, puis, après des études d'histoire, au Conservatoire national d'art dramatique de Paris (de 1987 à 1990), Stanislas Nordey est invité dès 1992 en résidence au Théâtre Gérard-Philipe/Centre dramatique national de Saint-Denis, dirigé par Jean-Claude Fall. À son actif : deux mises en scène, celle de La Dispute de Marivaux, remarquée lors du festival d'Avignon Off en 1988, et celle de Bête de style de Pier Paolo Pasolini, accueillie à Saint-Denis pour l'ouverture de la résidence. Jean-Claude Fall mène alors une politique envers les jeunes compagnies ; Stanislas Nordey reprendra cette volonté de partage de l'outil de travail mais, pour l'heure, il profite de cet accueil pour se concentrer sur son travail de création.

La découverte de Pier Paolo Pasolini - sa liberté de parole sur les grands enjeux contemporains, son amour de la langue mais aussi son sens pédagogique - influence durablement l'esthétique de Stanislas Nordey, qui trouve dans l'œuvre de l'artiste italien des réponses à la question de l'engagement politique. Le souci des textes, qui n'est pas antithétique d'un travail sur le corps et la nudité, mêlé à l'influence du cinéma de Pasolini, dans lequel les gros plans, fixes, succèdent aux tableaux de groupe, conduit Nordey à un usage fréquent de la frontalité. Le texte proféré, adressé au public, exprime une conscience politique aiguisée.

La mise en scène de Vole mon dragon d'Hervé Guibert au Festival d'Avignon en 1994, spectacle de plus de sept heures, où des corps nus se heurtent à la nécessité de communiquer, où la langue des signes allie la problématique de la langue à celle du corps, marque les esprits : Stanislas Nordey est invité par Jean-Pierre Vincent à partager la direction artistique du Théâtre Nanterre-Amandiers. Le jeune metteur en scène poursuit le parcours d'un artiste aux multiples succès, apparemment davantage préoccupé par la recherche esthétique et formelle que par la question du public. Pourtant, cette expérience au sein du Centre dramatique de Nanterre le conduit à réfléchir aux liens entre le public et l'artiste, au sein d'une institution théâtrale issue de la décentralisation. Sa conception, influencée par le foisonnement formel des années 1970 et 1980 et sa rencontre avec Valérie Lang, qui lui apporte une connaissance intime des expériences suscitées par Jack Lang au festival de Nancy, se révèle marginale par rapport à celle des tenants du théâtre populaire. En effet, Stanislas Nordey revendique un théâtre de recherche, un théâtre d'expérimentation, où le geste premier se déploie vers la scène. La question du lien avec le public semble être traitée en dehors de la forme théâtrale qui s'épanouit sur le plateau. Les pionniers du théâtre populaire, au contraire, 
conditionnaient le lien avec le peuple à l'existence d'un répertoire spécifique, qu'il soit composé de créations, comme pour Romain Rolland, Maurice Pottecher, ou plus tardivement Jacques Copeau ${ }^{3}$, ou de chefsd'œuvre du répertoire, comme pour Jean Vilar ou Jean Dasté. Pourtant, le théâtre de Nordey, parce qu'il s'appuie sur le texte et intègre souvent un dispositif frontal, travaille la question de l'adresse au public, ce qui pourrait renvoyer à la nature « intrinsèquement politique » du théâtre ${ }^{4}$.

Le compagnonnage avec Jean-Pierre Vincent, professeur apprécié au Conservatoire, débouche sur des divergences. Stanislas Nordey, épaulé par Valérie Lang qui n'a pas oublié la difficile expérience de son père à Chaillot lorsqu'il fut contraint de démissionner en 1974, éprouve la rigidité de l'institution théâtrale, marquée par le repli sur soi, l'indifférence aux publics de proximité et la course aux succès, par l'adoption des « recettes » du théâtre privé. Au fur et à mesure, et malgré le confort des conditions de création, Stanislas Nordey se démarque de son "père » pour, finalement, critiquer sévèrement la génération issue de mai 1968, qui refuse de partager les institutions dont ils ont eux-mêmes hérité. Alors que les années 1970 voient l'avènement de la toute-puissance du metteur en scène, Stanislas Nordey, lui, valorise l'acteur et privilégie la sobriété du dispositif scénique pour mieux entendre le texte. Il définit alors la mise en scène comme "art de l'effacement $»^{5}$. Le jugement de Nordey sur Mai 1968 et la déclaration de Villeurbanne, qui a donné lieu au slogan du "pouvoir au créateur », apparait sévère : «Mai 1968 n’était rien d'autre qu'une révolution de bourgeois. Les gens en place dans les théâtres sont pour moi les purs produits de cette bourgeoisie $»^{6}$.

Alors que Stanislas Nordey profite de la manne financière des années Lang, alors qu'il bénéficie d'une reconnaissance précoce, il fissure la filiation parfois établie entre Mai 1968 et Mai 1981. En pénétrant dans l'institution, il introduit un germe de subversion.

\section{En quête d’héritages}

Si la nomination de Stanislas Nordey à la tête d'un centre dramatique ne peut apparaître comme une véritable surprise, la reconnaissance médiatique compensant la jeunesse, ses prises de position et l'orientation du projet culturel pourraient, au premier abord, provoquer quelque étonnement.

Le premier acte du directeur s'inscrit dans la tradition théâtrale des manifestes. La publication « Pour un théâtre citoyen », qui s'ouvre par une date, «Année 1998 », semble relever d'une volonté de fonder une expérience, au sens d'ouvrir une nouvelle ère, qui viendrait balayer les expériences précédentes. Il s'agit « d'accomplir une révolution », c'est-àdire « de tourner sur soi-même pour porter un regard clair sur tout ce et 
tous ceux qui nous entourent». Le projet se décline au travers d'un ensemble de dispositifs de relations avec le public, visant à faciliter sa venue et l'ouverture du théâtre vers le territoire le plus proche. La mesure la plus emblématique concerne l'établissement d'un tarif unique de 50 francs (environ 8 euros) et la suppression de toutes les exonérations. Le souci de partage de l'outil de travail, la multiplication des propositions artistiques, dans et en dehors du théâtre, ainsi que la priorité donnée au répertoire contemporain complètent le projet.

Pourtant, malgré l'ambition de renouveau qui commande la rédaction du texte, Stanislas Nordey et son équipe mêlent à leur profession de foi des citations extraites des textes-manifestes de leurs prédécesseurs, issues de l'anthologie publiée par Jean-François Dusigne ${ }^{7}$. Sont ainsi cités des extraits du "Manifeste du Théâtre d'Action d'Art» de Louis Jouvet (1909), du «Discours sur l'acteur» de Max Reinhardt (texte sans date, publié en 1950, mais datant du début du XXème siècle), d'un entretien avec Ariane Mnouchkine sur «l'artisanat de l'art» (1984) et, enfin, de l'«Essai de rénovation dramatique» de Jacques Copeau publié à La Nouvelle Revue Française lors de l'ouverture du Théâtre du Vieux-Colombier en 1913. Le choix des artistes renvoie aux fondements du théâtre public tel qu'il émerge en France après la Seconde Guerre mondiale et qui se caractérise par l'association étroite, vécue désormais sans contradiction, entre le théâtre populaire et le théâtre d'art, entendu comme théâtre de haute culture, c'est-à-dire entre l'utopie de toucher un large public et la volonté de proposer aux spectateurs des œuvres de haute tenue artistique et réalisées sans concession mercantile ou commerciale. Comment, alors, expliquer ce qui apparaît comme une concession à l'air du temps : l'utilisation de l'adjectif « citoyen» en lieu et place de l'adjectif « populaire »? Refus d'utiliser un vocable trop facilement identifié à l'idéologie communiste? Volonté de rebaptiser un héritage pour éviter le sentiment mortifère d'un retour complet vers le passé ? Adaptation d'un projet à la société de la fin du XXème siècle? Cette dernière hypothèse inscrit le projet de Nordey dans un contexte social marqué par la montée du Front National, concomitamment à celle de l'abstention électorale, par l'émergence des questions urbaines et par la difficulté de réduire la question sociale à la seule lutte des classes, voire à l'existence même des classes sociales. Il ne s'agit plus, comme pour Jean Vilar, de constituer une communauté assemblée dans l'espace théâtral, en instaurant une « fiction d'égalité » le temps de la représentation ${ }^{8}$. Pour Nordey, le spectateur doit prendre part à la représentation, mais, plus encore, le théâtre doit éveiller sa conscience de citoyen, ce qui pourrait justifier la dénomination de "théâtre citoyen ». Stanislas Nordey s’inscrirait alors dans la société de son temps qui, depuis la fin des années 1960, valorise la place et le rôle de l'individu au sein de l'espace public, tout en se méfiant de toute empreinte idéologique.

En définissant le théâtre comme engagement, comme communauté et comme "poésie dialoguée », Stanislas Nordey reprend les fondamentaux du théâtre populaire. En effet, la conception du théâtre comme lieu de l'engagement personnel, justifiant l'effort et le sacrifice de chacun, sans 
concession ni compromis, forme l'axe éthique du projet de Stanislas Nordey. L'équipe du TGP adhère à cette notion de «théâtre comme vocation ", ce qui induit une réforme des relations salariales, mais risque, sur le long terme, d'épuiser l'énergie des salariés. Cet engagement pour l'art trouve sa réalisation concrète au sein d'une troupe. Nordey a commencé sa formation d'acteur au sein de l'atelier de sa mère, où il est « celui qui regarde les autres $\gg$ '. Un premier groupe d'acteurs s'est constitué autour de lui, bientôt complété par des camarades du Conservatoire. Nordey impose sa troupe quand il rejoint le Théâtre de Nanterre-Amandiers. C'est également un collectif qui signe le manifeste du TGP.

Pourtant, le rapport de Nordey à la troupe - comme d'ailleurs son rapport au pouvoir - semble plus complexe que la simple posture de chef de troupe, assumant son rôle et sa fonction d'autorité ${ }^{10}$. Stanislas Nordey, lui-même comédien, indique qu'en tant que metteur en scène, il a « besoin de préserver [sa] libido ${ }^{11}$, en s'autorisant à désirer fortement un acteur particulier. Il a également une conception de l'acteur qui rend fragile la notion de troupe et de communauté. Pour lui, l'acteur doit être force de proposition, rejoignant ainsi la recherche de Max Reinhardt. C'est pourquoi plusieurs de ses acteurs, comme Frédéric Fisbach ou Laurent Sauvage, sont devenus eux-mêmes metteurs en scène. Nordey justifie cette volonté d'ouverture, cette "permissivité », par la crainte de mettre les comédiens dans une situation telle qu'ils soient obligés de tuer le maître. Dans le projet de Saint-Denis, se joue un nouveau rapport au pouvoir : le metteur en scène reconnu accepte de se mettre en retrait pour accueillir un grand nombre de compagnies. Ce « dessaisissement du pouvoir », subversif dans le fonctionnement institutionnel du théâtre, impose une conception égalitaire du travail au sein d'une troupe. Ce refus de la carrière - Nordey aurait pu prendre la succession de Jean-Pierre Vincent - illustre également l'idéal de chef de troupe tel que Jeanne Laurent le prône et qu'incarnent les animateurs de la décentralisation dramatique qui, attachés à un lieu, refusent la «valse des directeurs » en 197412.

L'amour de la langue, le souci du texte doit s'analyser en lien avec le refus d'un pouvoir autoritaire. Alors que le théâtre évolue vers les " écritures de plateau $»^{13}$, Stanislas Nordey, lui, affiche un rapport somme toute traditionnel à la mise en scène : "Je considère que le théâtre, c'est faire passer des textes, transmettre une langue; si je dois faire œuvre comme créateur, je préfère travailler sur l'image ou sur les arts plastiques. Je ne veux pas mélanger mon engagement dans un théâtre de la langue, de la parole, avec les arts plastiques. J'aime trop les structures dramaturgiques, je préfère m'inscrire dans une tradition théâtrale, qui va d'Eschyle, en passant par Racine et Shakespeare, jusqu'à aujourd'hui. La théâtralité nécessite une trame, une fable, des personnages, une histoire $\gg^{14}$. Comme chez Jacques Copeau, la primauté du verbe se marie à la sobriété de la mise en scène, la finesse de la direction d'acteurs et le dénuement du plateau; les accessoires inutiles ne doivent pas perturber l'attention du public. 
Pourtant, malgré l'enthousiasme de la troupe de Nordey et les efforts de son équipe, le projet de «théâtre citoyen » ne survit que trois ans.

\section{Money versus utopie}

En octobre 1999, le ministère de la Culture rend publique la «crise financière grave que connaît le Théâtre Gérard-Philipe de Saint-Denis dirigé par Stanislas Nordey $»^{15}$. Le TGP devient l'objet de vifs débats dans le milieu théâtral. Les partenaires publics reprochent à la direction du théâtre un déficit de près de dix millions de francs (environ 1476000 euros) et lui demandent de le réduire de six millions de francs (environ 886000 euros) à l'issue de son mandat triennal, fin décembre 2000. Le plan d'apurement du déficit prévoit une baisse du volume d'activités, le recrutement d'un nouvel administrateur et la mise en place d'un comité de suivi. Des hommes de théâtre, mais aussi la presse culturelle, s'engagent publiquement en faveur du projet du TGP (Jean-Pierre Vincent et François Le Pillouër, notamment). D’autres, au contraire, se désolidarisent du jeune metteur en scène. Bernard Sobel en appelle à la responsabilité du directeur d'établissement public: "Dans le monde qui est le nôtre, cette pratique [théâtrale] (tant mieux s'il lui arrive de mériter d'être qualifiée d'art) est fragile et réclame, de la part de ceux qui acceptent de partager avec l'État la gestion de l'argent public, le plus grand sens des responsabilités $»^{16}$. Fin 2000, Stanislas Nordey quitte ses fonctions, après avoir refusé de renouveler son contrat.

La «crise » du TGP prend une ampleur toute particulière au moment où le ministère de la Culture, dirigé depuis 1998 par Catherine Trautmann, cherche à résoudre le malaise persistant du théâtre public. L'absence d'engagement du ministère paraît alors étonnante puisque le projet de Stanislas Nordey d'un «théâtre citoyen» répond aux nouvelles préoccupations de la ministre socialiste, qui souhaite renforcer la démocratisation de la culture. En imposant aux théâtres nationaux une soirée au tarif unique de 50 francs (8 euros), le ministère entend lutter contre les freins économiques de la venue au théâtre. Par ailleurs, la «Charte des missions de service public pour le spectacle vivant» exige des artistes une responsabilité accrue vis-à-vis des publics, des territoires et des jeunes compagnies. Si le projet de Stanislas Nordey est inattaquable sur ce premier point, l'évaluation des établissements sur le respect des contraintes budgétaires, incluse dans la charte, semble conduire le ministère, en dehors de raisons politiciennes ${ }^{17}$, à sanctionner la gestion déficitaire du TGP.

L'échec financier de l'expérience de Saint-Denis témoigne de la complexité de la position de Stanislas Nordey face à l'institution. Malgré son inscription dans le paysage institutionnel du théâtre public, l'artiste affirme la primauté de l'utopie et la grandeur d'un projet humaniste, contre les réalités financières ou matérielles. En cela, Stanislas Nordey 
rejoindrait les premières aventures du théâtre populaire, telles que la mémoire collective du milieu théâtral a pu les (re)construire. L'histoire du théâtre populaire charrie, en effet, une multitude d'exemples hérö̈ques, qui forgent peu à peu le mythe d'un théâtre du dénuement, où la solidarité de la communauté théâtrale pallie le manque de moyens matériels et financiers. L'image de la caravane de camions automobiles du Théâtre Ambulant, embourbée à quelques kilomètres de Paris, contraignant Firmin Gémier à stopper l'initiative après deux saisons de succès public (1911-1912), s'est transmise, malgré son inexactitude, dans la mémoire de l'histoire du théâtre ${ }^{18}$. Il est vrai, à y regarder de près, que les expériences innovantes du tournant du XIXème et du XXème siècles, en dehors du Théâtre du Peuple de Bussang, demeurent des expériences éphémères et fragiles ${ }^{19}$. La question financière constitue la pierre d'achoppement. Toutefois, il ne s'agit pas d'assimiler l'expérience du TGP à ces aventures pionnières, marquées par le mouvement syndical et/ou anarchiste: le Centre dramatique national de Saint-Denis appartient au réseau du théâtre public, impulsé après la Seconde Guerre mondiale par Jeanne Laurent, puis développé par André Malraux. Il bénéficie à ce titre de subventions publiques, contrairement aux premières initiatives de théâtre populaire. Il serait également tentant de rapprocher l'histoire de Stanislas Nordey au TGP de celle de Jean Vilar au Théâtre National Populaire (TNP). Malgré le succès public, Jean Vilar peine à honorer son lourd cahier des charges, qui lui laisse une marge de manœuvre étroite pour la production. Responsable sur ses propres deniers, Jean Vilar veille méticuleusement à l'équilibre budgétaire, ce qui lui interdit de manier le déficit comme arme de négociation avec les partenaires publics. A contrario, l'irresponsabilité financière des directeurs d'établissements publics contribue à expliquer l'assimilation du déficit à un argument de négociation politique. En effet, Nordey justifie une large part du déficit par l'inadéquation entre les missions validées par l'État et les collectivités et les subventions allouées.

Au final, le déficit du TGP pourrait s'apparenter à l'ambition de Firmin Gémier d'accorder sa parole aux actes, telle que Jacques Copeau la décrit : «La contribution de Gémier n’aurait pas été ce qu'elle a été s'il se fût borné à la théorie. Tout ce que lui inspirait son imagination, il a essayé de le réaliser $»^{20}$.

Parler de filiation ou d'héritage implique nécessairement la notion de transmission. S'interroger sur sa propre capacité à transmettre, envisagée sous le modèle du don, forme alors la suite logique d'un parcours marqué par la revendication de multiples héritages. Nommé par François Le Pillouër, en juin 2000, responsable pédagogique de l’École supérieure du Théâtre national de Bretagne (TNB), Stanislas Nordey met en œuvre depuis sept ans une démarche pédagogique qui prolonge son geste théâtral, de metteur en scène, mais aussi de comédien. Comme pour Jacques Copeau, la direction d'acteurs s'apparente à une rencontre où la transmission en face à face, du metteur en scène au comédien, par le verbe et le corps, conduit à un chemin commun, fait de partage et de découvertes réciproques. Stanislas Nordey entend faire vivre la poursuite d'un continuum générationnel du théâtre populaire, en intégrant les évolu- 
tions du contexte socio-politique et les inflexions de l'esthétique théâtrale, ouvertes par le mouvement artistique et institutionnel issu des années 1980.

(1) Le Monde, 7 mars 1997.

(2) Citation extraite du livre d'Alfred Simon, Jean

Vilar, Besançon, La Manufacture, 1991, p. 36.

(3) Jacques Copeau essaiera, sans véritablement y parvenir, d'élaborer un répertoire de créations, adossé à la commedia dell'arte ; projet que reprendra, d'une certaine manière, Ariane Mnouchkine avec L'Áge d'or (1975).

(4) Voir Denis Guénoun, L'Exbibition des mots et autres idées du théâtre et de la philosophie, Belfort, Circé/Poche, 1998.

(5) Stanislas Nordey et Valérie Lang, entretiens avec Yan Ciret et Franck Laroze, Passions civiles, Genouilleux, La Passe du Vent, 2000, p. 84.

(6) Ibid., p. 78.

(7) Jean-François Dusigne, Du théâtre d'art à l'art du théatre. Anthologie des textes fondateurs, Paris, Éditions Théâtrales, 1997.

(8) Laurent Fleury, "Abolition des classes sociales ou production d'une fiction d'égalité ? La réalisation d'un espace utopique au T.N.P. de Vilar ", in Jean-Noël Chopart et Claude Martin (dir.), Que reste-t-il des classes sociales? Lien social et politique, Rennes, Éditions de l'École Nationale de Santé Publique, 2004, p. 121-136.

(9) Stanislas Nordey et Valérie Lang, entretiens avec Yan Ciret et Franck Laroze, op. cit., p. 113. (10) Voir l'article de Libération du 21 novembre 1995, titré «L'année passée au Théâtre de Nanterre laisse le jeune metteur en scène songeur ", dans lequel Stanislas Nordey exprime ses doutes quant à l'officialisation d'un groupe d'acteurs en troupe.

(11) Stanislas Nordey et Valérie Lang, entretiens avec Yan Ciret et Franck Laroze, op. cit., p.115. (12) Voir Marion Denizot, «La menace des ambitions personnelles », in Jeanne Laurent. Une fondatrice du service public pour la culture. 1946-1952, Paris, La Documentation Française, 2005, p. 135-137.

(13) Voir Bruno Tackels, "Écrivains de plateau », in Les Castellucci, Besançon, Les Solitaires Intempestifs, 2005, p. 13-22.

(14) Stanislas Nordey et Valérie Lang, entretiens avec Yan Ciret et Franck Laroze, op. cit., p. 111.

(15) Communiqué du ministère de la Culture et de la Communication, 11 octobre 1999.

(16) Bernard Sobel, «Le gâchis Nordey », Le Monde, 15 novembre 1999.

(17) Stanislas Nordey avance aujourd'hui trois raisons qui expliqueraient l'absence de soutien dont il aurait été victime : l'inimitié de Catherine Trautmann qu'il avait prise publiquement à partie lors de sa nomination, alors que le collectif budgétaire décidé par le précédent gouvernement était maintenu ; son inscription sur la liste du Parti Communiste dirigée par Robert Hue, ennemi direct de Patrick Braouzec alors maire de Saint-Denis et, enfin, ses liens privés avec Valérie Lang, fille de l'ancien ministre de la Culture. Entretien Marion Denizot / Stanislas Nordey, 31 mai 2007.

(18) Voir Catherine Faivre-Zellner, Firmin Gémier, béraut du théâtre populaire, Rennes, Presses Universitaires de Rennes, 2006.

(19) Citons, pour exemples, le Théâtre Civique de Louis Lumet (1897-1899), le Théâtre du Peuple d'Henri Dargel (1899-1901), le Théâtre Populaire de Belleville d'Émile Berny (1903-1905), le Théâtre du Peuple d'Henri Beaulieu (1903-1904). (20) Jacques Copeau, Le Théatre populaire, Paris, Presses Universitaires de France, coll. « Bibliothèque du peuple », 1941, p. 23. 